calcitonin levels. In $88 \%$ of patients, plasma calcitonin was undetectable, indicating no evidence of disease. Postoperative elevation of plasma calcitonin was recorded in $21 \%$ of children who were at least 8 years old at the time of surgery, whereas levels of this tumor marker remained undetectable in all patients who underwent surgery aged 7 years or younger $(P=0.03)$.

The authors concluded that total thyroidectomy is an effective treatment for children who are at risk of developing MTC, but that the timing of the procedure is crucial.

Alexandra King

Original article Skinner MA et al. (2005) Prophylactic thyroidectomy in multiple endocrine neoplasia type 2A. N Engl J Med 353: 1105-1113

\section{Gene expression predicts chemotherapy response in advanced breast cancer}

Studies have shown that DNA microassay-based gene expression profiling of tumor tissue gives prognostic and predictive information that cannot be provided by traditional methods. Recent work using real time reverse transcription polymerase chain reaction (RT-PCR) to quantify gene expression in formalin-fixed, paraffin-embedded (FPE) tumor specimens identified a 21-gene Recurrence Score (RS) assay, which quantifies the risk of distant recurrence in breast cancer patients treated with adjuvant tamoxifen. Using these methods, Gianni and colleagues examined the correlation between RS and pathological complete response ( $\mathrm{pCR}$ ) to preoperative chemotherapy (paclitaxel and doxorubicin), and analyzed the association between gene expression and pCR.

The expression of 384 genes (including the 21-gene RS panel) was quantified by RT-PCR in 89 FPE bioptic specimens of locally advanced breast cancer. Eighty-six genes were found to correlate with pCR; pCR was more likely with higher expression of proliferation-related and immune-related genes, and lower expression of estrogen-receptor-related genes. In an independent cohort of 82 women who were treated in another hospital and whose tumors were profiled by microarray, 24 genes correlated with pCR with $P<0.05$ and 32 showed correlation with $P<0.1$. There was a positive association between likelihood of pCR and RS, suggesting that patients at the greatest risk of recurrence are more likely to benefit from chemotherapy.

The authors conclude that the ability to predict response to therapy using FPE tumor tissue would allow the tailoring of chemotherapy to individual patients.

Carol Lovegrove

Original article Gianni L et al. (2005) Gene expression profiles in paraffin-embedded core biopsy tissue predict response to chemotherapy in women with locally advanced breast cancer. J Clin Oncol 23: 7265-7277

\section{Ovoid shields reduce radiation dose to the rectum during ICRT for cervical cancer}

Fletcher Suit Delclos (FSD) (Nucletron Trading, Leersum, The Netherlands) ovoid shields were developed with the aim of protecting nontarget tissues in patients undergoing intracavitary radiotherapy (ICRT). Gifford and colleagues at the MD Anderson Cancer Center, Houston, TX, have recently assessed the effectiveness of FSD ovoid shields in reducing radiation dose to the rectum and bladder in 12 patients undergoing low-dose-rate ICRT for carcinoma of the uterine cervix.

The effect of ovoid shields on the radiation dose distributions in patient CT scans was determined using the Monte Carlo dosimetry method and cumulative dose-difference surface histograms. Dose-volume histograms were used to calculate the minimum radiation doses to the $2 \mathrm{~cm}^{3}$ and $5 \mathrm{~cm}^{3}$ of bladder $\left(D_{\mathrm{BV} 2}\right.$ and $\left.\mathrm{D}_{\mathrm{BV} 5}\right)$ and rectum $\left(D_{R V 2}\right.$ and $\left.D_{R V 5}\right)$ receiving the highest dose.

Although shielding did not significantly reduce the radiation dose to the bladder, rectal tissue was shown to be protected by the shields, with $10.1 \%$ and $11.1 \%$ dose reductions to $D_{R V 2}$ and $D_{\mathrm{RV} 5}$, respectively. The average dose reduction to $5 \%$ of the rectal surface area, as calculated by the cumulative dose-difference surface histograms, was 195 cGy, with a 405 cGy reduction recorded in one patient. The authors conclude that FSD ovoid shields are beneficial in protecting rectal tissue from radiation in patients receiving ICRT for cervical cancer, and should be included in 3D CT-based dosimetry.

Alexandra King

Original article Gifford KA et al. (2005) A three-

dimensional computed tomography-assisted Monte Carlo evaluation of ovoid shielding on the dose to the bladder and rectum in intracavity radiotherapy for cervical cancer. In $J$ Radiat Oncol Biol Phys 63: 615-621 